\title{
Mejora de constructos físicos en adultos mayores de la zona norte de México Improvement of physical fitness components in older adults from northern Mexico
}

Carlos Humberto Castañeda-Lechuga, Sergio Macias-Ruvalcaba, Jesús José Gallegos-Sánchez, Mario Alberto VillarrealAngeles

Universidad Juarez Estado de Durango (México)

Resumen. En México las tendencias demográficas en la población adulta registran un incremento eminente, y sin embargo son menos frecuentes los estudios y propuestas para los adultos mayores. Este estudio busca evidenciar el progreso y mejora de la condición física saludable, a través de un esquema basado en la actividad física en los adultos mayores, en el cual el Objetivo planteado fue analizar y estudiar los efectos de un programa de acondicionamiento físico que beneficie la salud y calidad de vida en el adulto mayor en la Metodología se aplicó un diseño cuasi-experimental de (pre-test) y (pos-test), en un solo grupo experimental (GE), mediante la intervención de un programa de actividad física adecuado a las particularidades de la edad y las condiciones físicas de los participantes, con 3 mediciones de la variable en sucesión cronológica, como instrumento de evaluación se utilizó el Senior Fitness Test (Rikli \& Jones, 2012) como principales Resultados se encontró que para todas las variables medidas, existe una interacción estadísticamente significativa con valores menores a ( $\mathrm{p} \mathrm{d»} \mathrm{.05)} \mathrm{en} \mathrm{las} \mathrm{variables} \mathrm{de} \mathrm{Agilidad,} \mathrm{Equilibrio,} \mathrm{Flexibilidad} \mathrm{en} \mathrm{miembros} \mathrm{inferiores,} \mathrm{Flexibilidad} \mathrm{en} \mathrm{miembros}$ superiores, Fuerza en extremidades superiores, Resistencia y Velocidad de Marcha Concluyendo que el programa de actividad física planificado durante 12 semanas, en tres sesiones semanales con una duración de 60 minutos por sesión, origina cambios positivos en variables físicas de adultos mayores de la zona norte de México.

Palabras Clave: Actividad Física, Adulto Mayor, Calidad de Vida, Entrenamiento.

Abstract. In Mexico, demographic trends in adulthood have registered a noticeable increase, yet studies and programs for older adults are less frequent. This study seeks to demonstrate the progress and improvement of healthy physical condition through physical activity in older adults. The objective was to analyze and study the effects of a physical conditioning program that benefits health and quality of life in elderly. The Methodology was based on a quasi-experimental pre-post-test design, using a single experimental group (GE) receiving an intervention program on physical activity adapted to age and physical condition peculiarities of the participants, with 3 measurement sessions. The evaluation instrument was represented by the Senior Fitness Test (Rikli \& Jones, 2012). Among the main results, statistically significant interaction was found for all the measured variables, with values lower than ( $\mathrm{d}$ » .05) in the variables of Agility, Balance, Flexibility in lower limbs, Flexibility in upper limbs, Strength in upper extremities, Resistance and Speed of March. As a conclusion, this 12-week program of physical activity, with a frequency of three 60-min sessions per week, determines positive changes in physical variables of older adults from the northern region of Mexico.

Keywords: Physical Activity, Older Adult, Quality of Life, Training.

\section{Introducción}

La inclusión e importancia que se les atribuye a los adultos mayores (AM) ha tenido cambios significativos, esto ha estado condicionado por múltiples factores ya que el envejecimiento es un proceso natural acompañado de un deterioro estructural y funcional con aparente disminución en los diferentes dominios, lo que afecta la autonomía personal y calidad de vida para los adultos mayores. (Martínez, Prada, Díaz \& Martínez-Patiño, 2011).

El crecimiento poblacional de los AM, junto con un patrón de desarrollo económico lleva una tendencia hacia desvalorizar sistemas tradicionales y solidarios de atención en salud en un bien de consumo, todo esto desarrolla escenarios no muy alentador para los AM (Aranibar, 2001).

En el año 2000 habitaban en el mundo 600 millones de personas mayores de 60 años y para el 2009 habían sobrepasado los 700 millones y se proyecta que para el 2050 existirán 2000 millones de adultos mayores estas estadísticas muestran que la cifra se triplico en un lapso de 50 años. De este modo se calcula que hacia el 2050 la población de AM seguirá creciendo a gran escala en relación con el resto de la población (Narlelis N. 2014).

El crecimiento de la población de AM se ha contrastado

Fecha recepción: 14-12-17. Fecha de aceptación: 03-08-19 Mario Alberto Villarreal-Angeles dr.villarreal82@gmail.com con el déficit de fertilidad y del crecimiento de las expectativas de vida de la población (Gonzalez \& Ham-Chande, 2007).

En otro sentido un problema más se encuentra en que según la Comisión Económica para América Latina y el Caribe (CEPAL), los países se pueden clasificar de acuerdo con la etapa en que se encuentran en su proceso de envejecimiento(OCDE, CAF, CEPAL 2018).

Según el Consejo Nacional de Población en México la esperanza de vida en el año 2000 era de 74 años pero ahora se estima que para el 2050 será de 80 años de edad, así como las estadísticas poblacionales de AM, en el año 2000 fue de 7\% de la población mexicana cuya cantidad se incrementara porcentualmente a 12.5\% en 2020 y 28.0\% en 2050 (BarrantesMonge, Garcia-Mayo, Gutierrez-Robledo, \& Miguel-Jaimes, 2007).

La vejez es un proceso de cambios determinados por factores fisiológicos, anatómicos, psicológicos y sociales y con el aumento de la edad, disminuye la capacidad de los AM hacia el rendimiento de estos factores (Hoppeler, Perrig, Buschkühl, Lippuner, Steiner, Breil, Däpp, \& Müller. 2009)

Según (Fulop et al., 2010) El proceso de envejecimiento se establece como un fenómeno natural que presenta una disminución de las capacidades fisiológicas, declinando progresivamente los órganos y sistemas.

Habitualmente, la edad cronológica ha establecido el parámetro que decreta el inicio de la ancianidad, sin embargo no decreta lo eficaz, capaz que podría llegar a ser un AM, en 
este sentido para mejorar las condiciones de vida en la edad avanzada, las personas mayores deben entrenar constantemente su fuerza muscular (Hoppeler et al. 2009).

Investigaciones refieren que la etapa de la vida del AM es cuando existen mayores riesgos de morbilidad y tasas de mortalidad por enfermedades crónico no trasmisibles (ECNT), siendo entonces esta una etapa de la vida del ser humano con una necesidad mayor en atención de salud, impactando de manera directa la calidad de vida (Thompson, Zack, Krahn, Andresen, \& Barile, 2012).

Sin embargo, el hecho de que una gran parte de las personas mayores padezca una falta de actividad física llevando una vida sedentaria y con esto una falta de calidad de vida es generalmente conocido (Stuck, Elkuch, Dapp, Anders, Iliffe \& Swift. 2002) con esto presentando mayores problemas para desempeñar sus actividades de la vida diaria, (Serrano-Sanchez, Lera-Navarro, \& Espino-Torón, 2013), disminuyendo su autonomía funcional y su capacidad de realizar acciones motrices de manera independiente, sin auxilios de personas y/o aparatos (Martinho et al., 2013).

Estos problemas motrices se relacionan con el deterioro funcional asociado a la presencia de discapacidad motriz la cual afecta al 56\% de los hombres y 62\% de las mujeres mayores de 60 años de edad (Barrantes-Monge et al., 2007).

Además que un estilo de vida poco activo o sedentario en AM predispone en una menor funcionalidad física como caminar, subir escaleras, levantarse de una silla de forma exitosa sin auxilio de una persona o aparato (de Noronha Ribeiro Daniel et al., 2011).

También es un hecho que la mayoría de los programas de actividades físicas dirigidos a los AM son enfocados en mejorar la capacidad de resistencia con un fuerte enfoque en el sistema cardiovascular (Hoppeler et al. 2009; Neuhauser, Thamm \& Ellert 2013). Sin embargo, con el aumento de la edad no solo disminuye la capacidad de resistencia sino también la fuerza muscular y la coordinación esto conllevan dificultad para cumplir con tareas de la vida diaria (Hoppeler et al. 2009).

En Europa Central las caídas en los AM son una de la razón más importante de visita en los hospitales y que posteriormente tiene consecuencias negativas, como largas visitas al hospital y tiempos de recuperación largos. (Hoppeler et al. 2009; Stuck, Elkuch, Dapp, Anders, Iliffe, \& Swift. 2002) en base en estos hechos, los programas orientados a los AM deben hacer énfasis en mejoran la mayor cantidad de capacidades físicas, pues son por lo tanto altamente recomendados (Hoppeler et al. 2009).

Es un derecho para el AM tener alternativas donde puedan realizar actividades sociales donde puedan desempeñar sus habilidades y creatividad. El entrenamiento puede incluirse como fuente de acción y elevar su calidad de vida mostrando posteriormente AM con buen bienestar físico, social y de salud. (Pereira, Fernández, Cruz \& Santiesteban. 2018; González \& Froment. 2018). Ninguna actividad debe tener un rango (estándar) de edades para su realización, y menos en la edad avanzada por sus múltiples déficits que se les presentan. Son necesarios un conjunto de actividades planificadas y especializadas para adultos mayores con la noción de una mejora en el desarrollo personal, psicológico y cambios fisiológicos presentes en este periodo de vejez.
Bajo este enfoque existen antecedentes de programas en atención al AM como el estudio de (Hall López, et al. 2017) denominado Programa de entrenamiento de hidrogimnasia sobre las capacidades físicas de adultas mayores donde el objetivo fue evaluar el efecto del programa de entrenamiento de hidrogimnasia sobre las capacidades físicas en $\mathrm{N}=26$ mujeres AM divididas en dos grupos (GE n=16) y $(\mathrm{GC} n=10)$ el instrumento de evaluación fue el Senior Fitness Test, la duración del programa fue de 12 semanas con una periodicidad de 5 veces por semana. Los resultados principales fueron que existió interacción significativa entre grupos y mediciones, para la prueba de resistencia de fuerza en extremidades inferiores ( $\mathrm{p}=0.052$ ), la prueba de agilidad de 2.4 metros (pd»0.01), las pruebas de capacidad aeróbica step test de 2 minutos ( $\mathrm{p}=0.02$ ) y caminar 6 minutos $(\mathrm{p}=0.50)$ concluyendo que se infiere que la hidrogimnasia en las adultas mayores participantes es eficaz en la mejora de las capacidades físicas principalmente aeróbicas, de resistencia a la fuerza y agilidad de miembros inferiores.

(Villarreal M., et al. 2016) en donde el propósito del estudio fue determinar el efecto de un programa de acondicionamiento físico basado en el método Pilates sobre variables del estado de ánimo en adultos mayores del Estado de Durango, México. El diseño fue un estudio experimental con un grupo control (GC) y un grupo experimental (GE) y mediciones iniciales (pre-test) y finales (post-test). Participaron 10 adultos mayores en cada grupo, edades entre los 60 y 80 años. La duración del programa fue de 12 semanas, con tres sesiones por semana de 50 min cada una. El instrumento de evaluación fue Profile of Mood States (POMS). Para el análisis estadístico se realizó una ANOVAde 2 × 2 . Como principales resultados encontraron una interacción significativa entre mediciones y grupos en las dimensiones de tensión ( $\mathrm{p}=$ $.001)$, cólera $(\mathrm{p}=.030)$, fatiga $(\mathrm{p}=.002)$ y el puntaje total ( $\mathrm{p} \mathrm{d}$ ) .0001). No se encontraron interacción significativa en la dimensión de depresión ( $\mathrm{p}=.065)$ ni vigor $(\mathrm{p}=.125)$. Concluyendo que un programa de acondicionamiento físico de 12 semanas basado en el método Pilates mejora algunas variables del estado de ánimo, las cuales pueden ser relevantes para el logro de la buena salud emocional en el adulto mayor.

\section{Metodología}

Se realizó un estudio de tipo cuasi-experimental, donde la VI fue manipulada para medir su efecto sobre la VD, con el objetivo de determinar el grado de cambio producido estableciendo una relación causa efecto, con muestra no probabilística seleccionada por conveniencia (Thomas, 2001), compuesta por AM mayores de 60 años conforme a la organización mundial de la salud (Gonzalez \& HamChande, 2007). Fue de corte Longitudinal pues existieron dos momentos de evaluación en el estudio el (pre-test) para conocer el estado inicial de la variable en estudio y (post-test) para contrastar los resultados entre estas dos mediciones (Campbell, Stanley, \& Gage, 1963).

\section{Participantes}

Se definió como población de estudio a los adultos Mayores entre 60 y 80 años. Se eligió una muestra voluntaria de 10 individuos, mediante los siguientes criterios de inclusión: 
a) Autosuficiencia de ejecutar actividades cotidianas, evitando la utilización de implementos de ayuda (e.g., bastón, andadera, silla de ruedas etc.) b) Condiciones morfológicas para la práctica de actividad física c) Compromiso de participar y permanecer en las sesiones del programa de AF. d) La no realización de otro tipo de AF durante la intervención. Fueron criterios de exclusión a) Afecciones que pudieran causar limitaciones para la realización de AF.

Para identificar los criterios de inclusión y exclusión se llevó a cabo una entrevista directa con cada prospecto y todos los sujetos participantes en el estudio proporcionaron el consentimiento informado para participar en el estudio que consistió en dar a conocer al sujeto de estudio el objetivo del proyecto, los procedimientos de evaluación, los posibles riesgos, beneficios y consecuencias, los procedimientos de emergencia y su anuencia de participación de carácter voluntario, de acuerdo con protocolos internacionales que protegen la integridad y los derechos de las personas que participan voluntariamente en investigaciones. Por las características de la metodología utilizada, la investigación no ocasiono riesgos físicos a los participantes y fueron respetadas todas las reglas éticas relacionadas con la confidencialidad de identidad e imagen personal. (Asociación Medica Mundial. 2008).

\section{Instrumento de medición}

Diversos estudios han relacionado la condición física con la capacidad funcional y con la calidad de vida relacionada con la salud (Villarreal M., et al. 2016), y se ha utilizado para medir los efectos de diversas intervenciones físicas en personas mayores. La evaluación de la condición física juega un papel importante en la promoción de la salud debido a que a través de ella, se puede identificar a las personas que poseen riesgo de contraer enfermedades, (Olivares, P.R.; 201x).

Es por ello que se seleccionó como instrumento para la medición el Senior Fitness Test (Rikli \& Jones, 2012), donde se considera que la condición física funcional tiene cinco dominios principales: composición corporal, fuerza muscular, resistencia cardiorrespiratoria, flexibilidad y equilibrio. Apoyan esta selección criterios prácticos como la factibilidad de los ejercicios para adultos mayores, la simplicidad de las instrucciones para su realización y la accesibilidad del equipamiento y también criterios estadísticos de validez y fiabilidad.

Para la prueba de 30 Second Chair stand que mide la fuerza de los miembros inferiores la fiabilidad es de $\mathrm{r}=.78$ para los hombres y .71 para las mujeres, para 30 Second Arm Curl que mide la fuerza de los miembros superiores la validez es de $r=.82$, para 6-Minute Walk que pone a prueba la resistencia aeróbica los criterios de validez son de $\mathrm{r}=.82$ para los hombres y $r=.71$ para las mujeres, para 2-Minute Step que evalúa resistencia aeróbica, la correlación con la prueba de Rockport es de $r=.73$, y de $r=.74$ con el rendimiento en caminadora (banda sin fin). Para la prueba Chair Sit-andReach que mide la flexibilidad de la parte inferior del cuerpo, la validez de criterio es de .76 para los hombres y .81 para las mujeres. Para la prueba Back Scratch que pone a prueba la flexibilidad de las extremidades superiores, la validez de constructo muestra que detecta reducción prevista en la fle- xibilidad del hombro en el 60, 70, y 80 años de los diferentes grupos de edad, dependiendo del nivel de actividad física. Para la prueba 8-Foot Up-and-Go que estima la agilidad, dinámica y equilibrio, la correlación es de $\mathrm{r}=.81$ con la escala Berg de Balance y de $r=.78$. Con el índice de Barthel de las Actividades de la Vida Diaria, los resultados indican que los valores de correlación test-retest para los ítems de SFT oscilaron .80 a .98, lo que indica la fiabilidad aceptable para todos los elementos de prueba (Bejarano-Roncancio, ArdilaGuzmán, \& Montaño-Rodríguez, 2014), demostradas en varias poblaciones, a partir de cuyas mediciones se han construidos los Rangos de Valores Estándares por grupos de edades entre 60 y 94 años, lo cual facilita el posterior procesamiento de los datos obtenidos.

\section{Procedimiento}

Se efectuó un estudio gradual comparativo en el (GE) con evaluación inicial para valorar el estado de las variables antes de iniciar el programa de AF. (pre-test) y evaluación final (post-test) después de 12 semanas de aplicación del programa de AF mediante el instrumento seleccionado Senior Fitness Test (Rikli \& Jones, 2012), para la VD. El programa de actividad física consistió en ejercicios basados en movimientos relacionados con las actividades de la vida cotidiana, por ejemplo pararse, caminar, estiramientos, respiraciones y sostener objetos medianamente pesados. Las sesiones se programaron para un periodo de tres meses (12 semanas), tres sesiones por semana y una duración de 60 minutos por sesión, las sesiones se llevaron a cabo mediante las recomendaciones de (Chodzko-Zajko et al., 2009) para la planificación de programas de actividad física en adultos mayores. a) calentamiento: mediante ejercicios de estiramiento y rangos de movilidad (10 min.) b) parte medular: realización del programa de AF (40 min.) c) cierre de clase: vuelta a un estado inicial mediante ejercicios de respiración y relajación las sesiones se llevaron a cabo en un área de $20 \mathrm{~m}^{2}$.

\section{Análisis estadístico de los datos}

Los análisis estadísticos se realizaron con el paquete estadístico IBM-SPSS Statistics, versión 21.0 (IBM Corporation, Armonk, New York, USA). Los valores descriptivos se presentan como la media y la desviación estándar $(\mathrm{M} \pm \mathrm{DE})$. Para los análisis inferenciales, se realizaron pruebas de análisis de varianza (ANOVA) Para ello se aplicó una ANOVA de una vía de medidas repetidas (1 grupo: experimental X 3 mediciones). Se encontraron interacciones significativas a un nivel de significación de p d» 5,05, se calcularon los análisis de efectos principales y análisis post hoc correspondientes.

\section{Resultados}

Para la variable Agilidad Se encontró una interacción estadísticamente significativa $(\mathrm{p}=.001)$ entre las mediciones pre y post. En donde la medición inicial $(\mathrm{M}=6.60 \pm 1.647)$, fue mayor que la medición final ( $M=5.40 \pm 1.265)$. Para la variable Equilibrio estático también se encontró una interacción estadísticamente significativa $(\mathrm{p}=.001)$ entre las mediciones pre y post, en donde la medición inicial ( $\mathrm{M}=35.5$ $\pm 19.208)$, fue menor que la medición final $(M=39.70 \pm 18.172)$. 
Se obtuvo una interacción estadísticamente significativa (p $=.0001$ ) entre las mediciones ante la variable de Flexibilidad extremidades inferiores pie derecho, en donde la medición inicial ( $M=-2.0200 \pm 3.001)$, fue menor que la medición final $(\mathrm{M}=-.3100 \pm 2.4528)$. Para la variable flexibilidad extremidades inferiores pie izquierdo se encontró una interacción estadísticamente significativa ( $\mathrm{p}=.0001)$ entre las mediciones del grupo experimental en donde la medición inicial ( $\mathrm{M}=$ $-6.07 \pm 5.43794)$, fue menor que la medición final ( $M=-3.6200$ $\pm 4.73634)$. Para la variable Flexibilidad extremidades superiores mano derecha se encontró una interacción estadísticamente significativa ( $\mathrm{p}=.001)$ entre las mediciones del grupo experimental en donde la medición inicial $(\mathrm{M}=$ $15.8800 \pm 11.89069)$, fue menor que la medición final $(\mathrm{M}=$ $13.8700 \pm 11.39406)$. También se encontró una interacción estadísticamente significativa ( $\mathrm{p}=$.0001) entre las mediciones del grupo experimental ante la variable de Flexibilidad extremidades superiores mano izquierda. En donde la medición inicial ( $M=-16.0200 \pm 11.06996)$, fue menor que la medición final $(M=-14.1700 \pm 10.59298)$. De igual manera para la variable de Fuerza en extremidades inferiores la interacción fue estadísticamente significativa $(\mathrm{p}=$.0001) entre las mediciones del grupo experimental, en donde la medición inicial $(\mathrm{M}=10.30 \pm 4.877)$, fue menor que la medición final $(\mathrm{M}=$ $12.60 \pm 5.481$ ). Por su parte ante la variable de Fuerza en extremidades superiores con brazo derecho se encontró una interacción estadísticamente significativa $(\mathrm{p}=.0001)$ entre las mediciones del grupo experimental, en donde la medición inicial ( $M=25.50 \pm 7.028)$, fue menor que la medición final (M $=28.20 \pm 7.177)$. De manera similar se encontró una interacción estadísticamente significativa ( $\mathrm{p}=.0001$ ) entre las mediciones del grupo experimental ante la variable de Fuerza en extremidades superiores con brazo izquierdo, en donde la me-

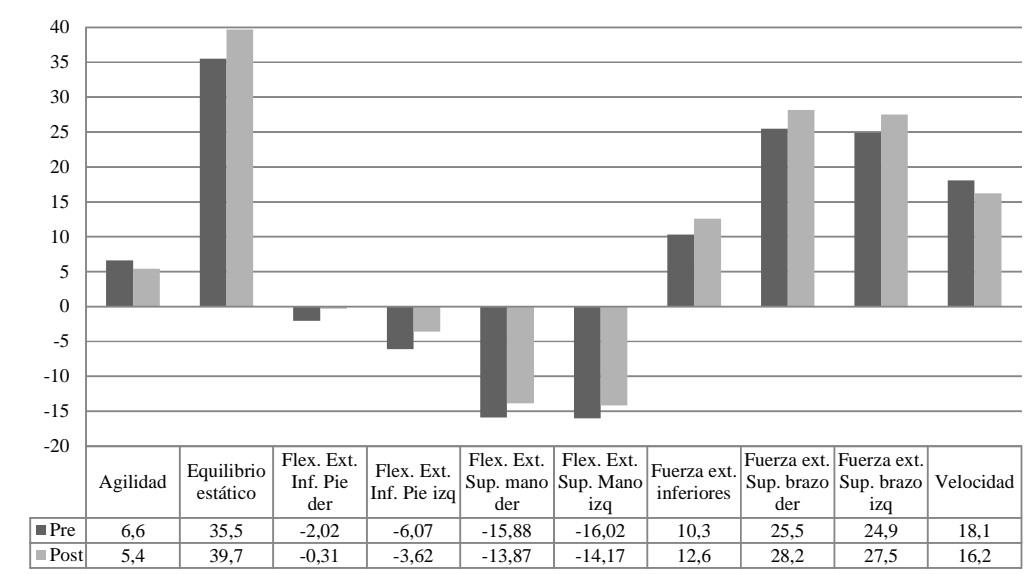

Figura 1. Resultados evaluaciones variables físicas pre - test y post - test.

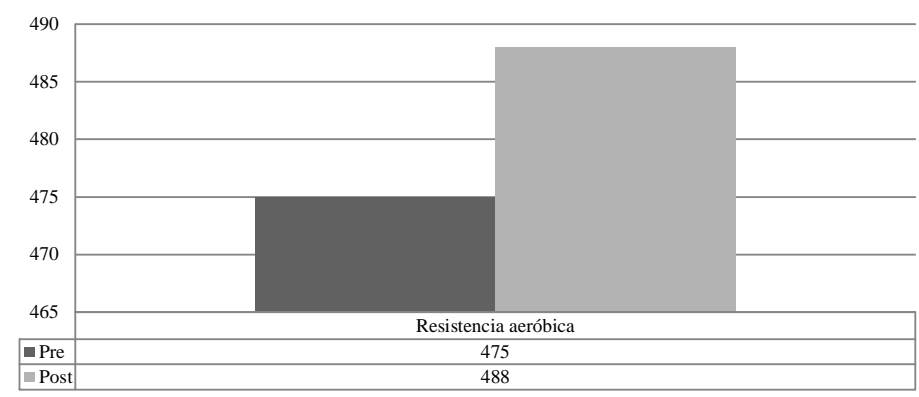

Figura 2. Resultados pre y post de la variable resistencia aeróbica. dición inicial $(M=24.90 \pm 6.100)$, fue menor que la medición final $(M=27.50 \pm 6.770)$. Tal como se observa en las variables anteriores, se encontró una interacción estadísticamente significativa $(\mathrm{p}=.0001)$ entre las mediciones del grupo experimental ante la variable de Resistencia aeróbica en donde la medición inicial $(\mathrm{M}=475.00 \pm 21.731)$, fue menor que la medición final $(\mathrm{M}=488.00 \pm 18.738)$. Y para la variable Velocidad de marcha se encontró una interacción estadísticamente significativa ( $\mathrm{p}=.0001$ ) entre las mediciones pre y post en donde la medición inicial $(\mathrm{M}=18.10 \pm 2.079)$, fue mayor que la medición final $(\mathrm{M}=16.20 \pm 1.398)$.

\section{Discusión}

El desenlace de este estudio muestra que la fuerza de miembros inferiores incremento relevantemente en la agrupación experimental. Estos resultados coinciden con los adquiridos de otros estudios (Fourie et al., 2012), donde se a descubierto que la ejecucion de actividad física durante ocho semanas es adecuado para ocasionar mejoras relativas en la fuerza y resistencia muscular de mujeres mayores de 60 años. En el caso de la fuerza de miembros superiores, se analizan resultados semejantes a los precedentes, en donde la agrupacion experimental tuvo avances significativos al concluir la operación llevando al mejoramiento de su fuerza. Como lo demuestra (Shea \& Moriello, 2014), quienes elaboraron un programa de Pilates durante 9 meses, existen mejoras significativas en el equilibrio, la fuerza de las extremidades inferiores, y la calidad de vida de un individuo con un derrame cerebral (accidente vascular cerebral, AVC). Para ambas variables.

Para la flexibilidad se muestra una mejora muy significativa. Este hallazgo concuerda con los estudios de (GonzalezRave, Sanchez-Gomez, \& Santos-Garcia, 2012), quienes realizaron un estudio para determinar la influencia de los 2 métodos de entrenamiento con estiramientos en el rango de movimiento con 54 participantes (39 mujeres y 15 hombres) entre las edades de 60 y 70 años durante un período de 13 semanas. Los primordiales descubrimientos indican que al utilizar entrenamientos de flexibilidad se adquirían incrementos en los rangos de movilidad de las articulaciones. Asimismo, el primordial descubrimiento fue la capacidad de las personas mayores físicamente activas con métodos de estiramientos incrementara el rango de movilidad. Para la flexibilidad en miembros superiores se analiza que la agrupación experimental tuvo mejora relevante al concluir la operación. Este descubrimiento coincide con otros (Billson et al., 2011), quienes realizaron una intervención con ejercicios de fuerza para conocer su repercusión en el rango de movilidad articular en 49 adultos entre 55 y 85 años de edad, quienes eran sedentarios y que fueron entrenados tres 
veces a la semana. El hallazgo del estudio fue que obtuvieron un aumento de la flexibilidad de hombros e isquiotibiales.

Para las variables Equilibrio, Flexibilidad, Fuerza y Resistencia los resultados del estudio son similares a los de (Vidarte Claros, J.A., Quintero Cruz, M. V., \& Herazo Beltrán, Y. 2012).en su estudio denominado Efectos del ejercicio físico en la condición física funcional y la estabilidad en adultos mayores, en donde el objetivo fue establecer los efectos del ejercicio físico sobre la condición física funcional y el riesgo de caídas en un grupo de adultos mayores realizaron un diseño de Estudio cuasi-experimental con grupo control y experimento en 38 adultos mayores de 60 años. Muestreo intencional, no probabilístico y aleatorio, el instrumento de evaluación utilizado fue el Senior Fitness Test, antes y después del programa de ejercicios, que tuvo una duración de 12 semanas sus principales resultados fueron en la fuerza de miembros superiores de 17,16 a 20,63 repeticiones; la flexibilidad de tren superior de -8,24 a -5,66 cm; una resistencia aeróbica de 70,32 a 83,47 pasos; el equilibrio de 13,95 a 15,26 puntos. Como conclusión Los resultados sugieren el aumento de la fuerza y flexibilidad de los miembros superiores, de la capacidad aeróbica, del equilibrio en un grupo de adultos mayores

Para la Agilidad, los resultados obtenidos de este estudio indican que mejoró significativamente. Estos resultados se asemejan a los de (Gildenhuys et al., 2013), quienes realizaron un estudio con 50 mujeres sedentarias, aparentemente sanas, mayores de 60 años, para determinar los efectos de un programa de entrenamiento de Pilates de ocho semanas, tres veces por semana, en la agilidad, movilidad funcional y VO2máx.

Fundamentado por esta gama de evidencias, (SolanoMora, 2013) finaliza y confirma que las actividades físicas provocan cambios favorables en los aspectos pragmáticos de los adultos mayores los cuales son concluyentes para su autonomía y calidad de vida.

\section{Conclusiones}

La finalidad de la presente indagación fue estudiar el resultado de un programa de actividad física mediante variables físicas en adultos mayores. La posibilidad general del estudio era encontrar un favorecimiento de las capacidades físicas de los adultos mayores por medio de la utilización del programa de acondicionamiento físico.

Se prescribe mejoramientos para los adultos mayores en aspectos físicos, los cuales son excelentes para consecución de buena salud, así mismo de su condición de vida. La fuerza, agilidad, resistencia aeróbica y flexibilidad mejoraron por medio del programa de actividad física, contribuyendo un efecto considerable de fortaleza, autosuficiencia pragmática, energía vitalidad para las actividades cotidianas. Para la población de adultos mayores puede ser factible la accesibilidad el programa de actividad física propuesto, ya que no es indispensable disponer de espacios grandes o equipamiento especializado y costoso para ejecutarse, es satisfactorio de simple práctica y realizado por los adultos mayores. De este modo, se concluye que el programa de actividades físicas, planificado durante 12 semanas, en tres sesiones semanales con una duración de 60 minutos por sesión, origina cambios positivos en variables físicas de adultos mayores de la zona norte de México.

\section{Referencias}

Asoociacion Medica Mundial (2008). Declaración de Helsinki de la Asociación Médica Mundial. Principios éticos para las investigaciones médicas en seres humanos. In Anales del Sistema Sanitario de Navarra (Vol. 24, No. 2, pp. 209-212).

Aranibar, P. (2001). Acercamiento conceptual a la situación del adulto mayor en América Latina. CEPAL.

Barrantes-Monge, M., García-Mayo, E. J., Gutiérrez-Robledo, L. M., \& Miguel-Jaimes, A. (2007). Functional dependence and chronic disease in older Mexicans. Salud publica de Mexico, 49(S4), 459-466. https:// w w w. m edig r a p h i c. c o m / c g i b in / n e w /

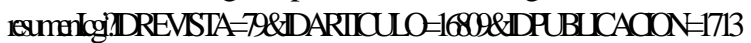

Bejarano-Roncancio, J. J., Ardila-Guzmán, L. S., \& MontañoRodríguez, A. (2014). Alimentación, nutrición y envejecimiento: un análisis desde el enfoque social de derechos. Revista de la Facultad de Medicina, 62(Supl 1), 73-79.

Billson, J. H., Cilliers, J. F., Pieterse, J. J., Shaw, B. S., Shaw, I., \& Toriola, A. L. (2011). Comparison of home-and gymnasium-based resistance training on flexibility in the elderly. South African Journal for Research in Sport, Physical Education and Recreation, 33(3), 1-9.

de Noronha Ribeiro Daniel, F., de Souza Vale, R. G., Giani, T. S., Bacellar, S., Escobar, T., Stoutenberg, M., \& Dantas, E. H. (2011). Correlation between static balance and functional autonomy in elderly women. Archives of Gerontology and Geriatrics, 52(1), 111-114. https:// doi.org/10.1016/j.archger.2010.02.011

Fulop, T., Larbi, A., Witkowski, J. M., McElhaney, J., Loeb, M., Mitnitski, A., \& Pawelec, G. (2010). Aging, frailty and age-related diseases. Biogerontology, 11(5), 547-563. https:/link.springer.com/article/10.1007/s10522-010-92872

Campbell, D. T., Stanley, J. C., \& Gage, N. L. (1963). Experimental and quasi-experimental designs for research: Houghton Mifflin Boston.

Chodzko-Zajko, W. J., Proctor, D. N., Fiatarone Singh, M. A., Minson, C. T., Nigg, C. R., Salem, G. J., \& Skinner, J. S. (2009). Exercise and Physical Activity for Older Adults. Medicine \& Science in Sports \& Exercise, 41(7), 15101530. https://www.bewegenismedicijn.nl/files/

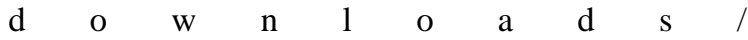
acsm_position_tand_exercise_and_physical_adivity_for_older_adults.pdf Fourie, M., Gildenhuys, G., Shaw, I., Shaw, B., Toriola, A., \& Goon, D. (2012). Effects of a mat Pilates programme on muscular strength and endurance in elderly women: exercise physiology. African Journal for Physical Health Education, Recreation and Dance, 18(2), 299-307.

Gildenhuys, G., Fourie, M., Shaw, I., Shaw, B., Toriola, A., \& Witthuhn, J. (2013). Evaluation of Pilates training on agility, functional mobility and Evaluation of Pilates training on agility, functional mobility and Cardiorespiratory fitness in elderly women: health and fitness. African Journal for Physical Health Education, Recreation and Dance, 19(2), 505-512.

González, A. J. G., \& Froment, F. (2018). Beneficios de la actividad física sobre la autoestima y la calidad de vida de personas mayores. Retos: nuevas tendencias en educa- 
ción física, deporte y recreación, (33), 3-9.

Gonzalez, C.A., \& Ham-Chande, R. (2007). Functionality and health: a typology of aging in Mexico. Salud Pública de México, 49(S4), 448-458. https://www.medigraphic.com/ cgi-bin/new/resumenI.cgi?IDARTICULO=16808

Gonzalez-Rave, J. M., Sanchez-Gomez, A., \& Santos-Garcia, D. J. (2012). Efficacy of two different stretch training programs (passive vs. proprioceptive neuromuscular facilitation) on shoulder and hip range of motion in older people. Journal of Strength \& Conditioning Research, 26(4), 1045-1051. doi: 10.1519/JSC.0b013e31822dd4dd

Hall López, J.A.; Ochoa Martínez, P.Y.; Alarcón Meza, E.I.; Moncada-Jiménez, J.A.; Garcia Bertruy, O. y Martin Dantas, E.H. (2017). Programa de entrenamiento de hidrogimnasia sobre las capacidades físicas de adultas mayores / Hydrogymnastics Training Program on Physical Fitness in Elderly Women. Revista Internacional de Medicina y Ciencias de la Actividad Física y el Deporte vol. 17 (66) pp. 283-298. DOI: https://doi.org/ 10.15366/rimcafd2017.66.005

Hoppeler, H., Perrig, W., Buschkühl, M., Lippuner, K., Steiner, R., Breil, F., Däpp, C., Müller, M. (2009). Seniorensport: intensiv bremsen statt langsam treten. Swiss Medical Forum (Vol. 9, No. 20, pp. 380-381). EMH Media.

Martínez Vidal, A., Prada Martínez, A., Díaz Pereira, M., \& Martínez-Patiño, M. (2011). Effect of a combined program of physical activity and intellectual activity in the cognitive functioning of the elderly. Journal of Human Sport and Exercise, 6(2), 462-473. http://dx.doi.org/ 10.4100/jhse.2011.62.27

Martinho, K. O., Dantas, E. H., Longo, G. Z., Ribeiro, A. Q., Pereira, E. T., Franco, F. S., Tinoco, A. L. (2013). Comparison of functional autonomy with associated sociodemographic factors, lifestyle, chronic diseases (CD) and neuropsychiatric factors in elderly patients with or without the metabolic syndrome (MS). Archives of gerontology and geriatrics, 57(2), 151-155. https:// doi.org/10.1016/j.archger.2013.04.005

Narledis, N. (2014). Capacidad funcional en adultos mayores que asisten a un programa de rehabilitación cardiaca. Ciencia e Innovación en Salud. https://doi.org/10.17081/ innosa.2.1.71

Neuhauser, H., Thamm, M., Ellert, U. (2013). Blutdruck in Deutschland 2008 - 2011. BundesgesundheitsblattGesundheitsforschung-Gesundheitsschutz, 56(5-6), 795801. https://doi.org/10.1007/s00103-013-1669-6

OCDE/CAF/CEPAL (2018), Perspectivas económicas de América Latina 2018: Repensando las instituciones para el desarrollo, Éditions OCDE, París. https:// repositorio.cepal.org/bitstream/handle/11362/43513/1/ LEO2018_es.pdf

Olivares, P.R.; Hernandez-Mocholi, M.; Merellano-Navarro, E.; Gusi, N. y Collado-Mateo, D. (201x). Análisis de la edad sobre la fiabilidad de pruebas fitness en mayores / Analysis of Age on Fitness Reliability in Elderly. Revista Internacional de Medicina y Ciencias de la Actividad Física y el Deporte vol. (*)pp. *. Http://cdeporte.rediris.es/ revista/

Pereira, L. G., Fernández, E. B., Cruz, M. G., \& Santiesteban, J. R. G. (2018). Programa de actividad física y su incidencia en la depresión y bienestar subjetivo de adultos mayores. Retos: nuevas tendencias en educación física, deporte y recreación, (33), 14-19.

Rikli, R. E., \& Jones, C. J. (2012). Senior Fitness Test Manual (Second ed.): Human Kinetics.

Serrano-Sanchez, J.A.; Lera-Navarro, A. y Espino-Torón, L. (2013) Actividad física y diferencias de fitness funcional y calidad de vida en hombres mayores / Physical activity and differences of functional fitness and quality of life in older males. Revista Internacional de Medicina y Ciencias de la Actividad Física y el Deporte, vol. 13 (49) pp. 87-105 Http://cdeporte.rediris.es/revista/revista49/ artactividad348.htm

Shea, S., \& Moriello, G. (2014). Feasibility and outcomes of a classical Pilates program on lower extremity strength, posture, balance, gait, and quality of life in someone with impairments due to a stroke. Journal of Bodywork and Movement Therapies, 18(3), 332-360. https://doi.org/ 10.1016/j.jbmt.2013.11.017

Solano-Mora, L. C. (2013). Efectos de la actividad física en la capacidad funcional de la persona adulta mayor In J. Moncada-Jiménez (Ed.), Efectos del ejercicio y el entrenamiento físico en el desarrollo, el rendimiento y la salud humana (pp. 525). San José, Costa Rica: Editorial de la Universidad de Costa Rica

Stuck, A.E., Elkuch, P., Dapp, U., Anders, J., Iliffe, S., Swift, C.G. (2002). Feasibility and yield of a self-administered questionnaire for health risk appraisal in older people in three European countries. Age and Ageing. 31(6), 463467. https://doi.org/10.1093/ageing/31.6.463

Thomas JR, Nelson JK, Silverman S, Silverman SJ. (2001) Research Methods in Physical Activity 6th. Ed. Champaign, Ilinois: Human Kinetics.

Thompson, W. W., Zack, M. M., Krahn, G. L., Andresen, E. M., \& Barile, J. P. (2012). Health-related quality of life among older adults with and without functional limitations. American journal of public health, 102(3), 496-502. doi: 10.2105/AJPH.2011.300500

Vidarte Claros, J.A., Quintero Cruz, M. V., \& Herazo Beltrán, Y. (2012). Efectos del ejercicio físico en la condición física funcional y la estabilidad en adultos mayores. Revista Hacia la Promoción de la Salud, 17(2), 79-90. https:// www.redalyc.org/pdf/3091/309126826006.pdf

Villarreal-Angeles, M. A., Jiménez, J. M., Sanchez, J. J. G., \& Ruiz-Juan, F. (2016). El efecto de un programa de ejercicios basado en Pilates sobre el estado de ánimo en adultos mayores Mexicanos (Effects of a Pilates-based exercise program on mood states in older adults in Mexico). Retos (30), 106-109. http://www.redalyc.org/ articulo.oa?id=345744747020

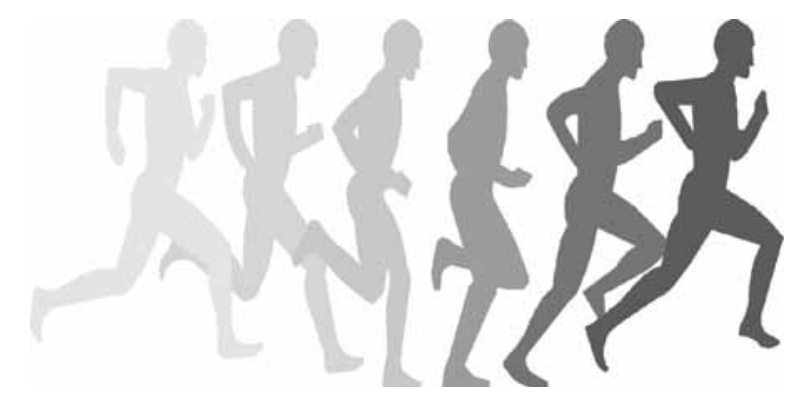

